\title{
Climate Change and the Expanding Global Reach of Dengue Fever - Warnings unheeded?
}

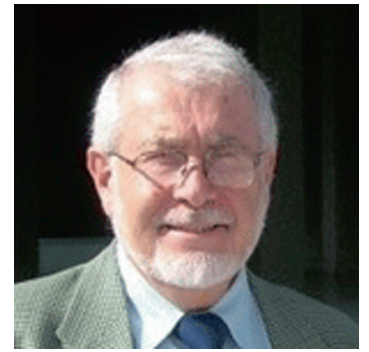

Dengue threatens the health of populations around the world due to the expanding distribution of the virus and its mosquito vectors, co-circulation of serotypes, and the emergence of more complex and life threatening forms of the disease in new areas. The US Centers for Disease Control and Prevention (CDC) in 2009 noted that dengue is now the world's leading arboviral disease, estimating 100 million cases of dengue fever annually, 250,000 of dengue haemorrhagic fever (DHF), and 25,000 deaths. ${ }^{1}$ Reported from $>100$ countries, 2.5 billion people now live in endemic areas.

But this is not really "new news": a decade earlier", mathematical models simulating climate change projected that rising global temperatures will increase the range of mosquitoes that transmit dengue. Johns Hopkins University researchers showed that epidemic potential increases with only a relatively small temperature rise: fewer mosquitoes are necessary to maintain or spread dengue in a vulnerable population. Predicted areas of encroachment were mostly temperate regions bordering on endemic zones, where humans and the mosquito Aedes aegypti (primary carrier), often co-exist, but where lower temperatures until then had supressed transmission. Global warming would not only increase the mosquito's range but also reduce the size of larva and, ultimately, adult size. Since smaller adults feed more frequently to develop eggs, warmer temperatures boost the frequency of double feeding and increase transmission potential. Also, virus incubation inside mosquitos is shortened at higher temperatures; this also can promote higher transmission rates.

The situation is becoming serious in many parts of the world. Because it now involves co-circulation of three serotypes, the potential for disease burden has been enhanced because a primary infection does not immunize against subsequent infection from another strain; to the contrary, it sets up a complex immune response that may result in more serious disease e.g., dengue haemorrhagic fever and dengue shock syndrome. Although there has been ample warning of this increasing threat, diminished priorities for health in many countries e.g., education and mosquito abatement, and deteriorating infrastructures e.g., drains and ditches (ideal for mosquito breeding), have weakened health protection in many settings. Increasingly it is also reported in travelers returning from infected areas. However, concerns for the impact on tourism have led paradoxically to reluctant recognition by the political establishment in some countries. This is self-defeating because ultimately any nation failing to take action will be more likely to become endemic. The major risk is of course for the people of the affected countries, especially those who live in heavily mosquito afflicted areas, most often the poor who typically have limited political representation. The adequacy of health care systems may not be sufficient to cope with epidemic surges. Clearly the appropriate response is to refocus on prevention.

Dengue fever is but one condition that is influenced adversely by climate change, for which genuine global, national and local leadership so far has been mostly deficient. From time to time, future editorials will address other issues related to this major challenge to the health of our planet.

\section{REFERENCES}

1. Wilder-Smith A, Chen LH, Massad E, Wilson ME. Threat of Dengue to Blood Safety in Dengue-Endemic Countries. Emerging Infectious Diseases. 2009 January; 15 (1):8-11.

2. Patz JA, Martens WJ, Focks DA, Jetten TH. Dengue fever epidemic potential as projected by general circulation models of global climate change. Environ Health Perspectives. 1998 March; 106(3):147-153.

Franklin White

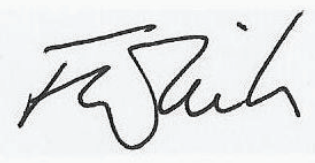

Associate Editor IJMEDPH

Submitted June 1, 2011 